\title{
THEORY OF COEXISTENCE OF SUPERCONDUCTIVITY AND FERROELECTRICITY: A DYNAMICAL SYMMETRY MODEL
}

\author{
Joseph L. Birman \\ Physics Department, The City College, CUNY, \\ Convent Ave. at 138 St, New York, NY 10031, USA \\ Meir Weger \\ The Racah Institute of Physics, \\ The Hebrew University Jerusalem ,Israel
}

(October 22, 2018)

\begin{abstract}
We propose and investigate a model for the coexistence of Superconductivity (SC) and Ferroelectricity (FE) based on the dynamical symmetries $s u(2)$ for the (pseudo-spin) SC sector , $h(4)$ for the (displaced oscillator) FE sector and $s u(2) \otimes h(4)$ for the composite system. We assume a minimal symmetryallowed coupling , and simplify the hamiltonian using a double mean-field approximation (DMFA). A variational coherent-state (VCS) trial wave-function is used for the ground state : the energy, and the relevant order parameters for SC and FE are obtained . For positive sign of the SC-FE coupling coefficient, a non-zero value of either order parameter can suppress the other one (FE polarization suppresses SC, and vice versa). This gives some support to "Matthias' Conjecture" [1964], that SC and FE tend to be mutually exclusive. For such a Ferroelectric Superconductor we predict: a) the SC gap $\Delta$ (and $T_{c}$ ) will increase with increasing applied pressure when pressure
\end{abstract}


quenches FE, as in many ferroelectrics and b) the FE polarization $|\vec{P}|$ will increase with increasing applied magnetic field up to $H_{c}$, which is equivalent to the prediction of a new type of Magneto-Electric Effect in a coexistent SC-FE material. Some discussion will be given of possible relation of these results to the cuprate superconductors .

Keywords: Superconductors, Ferroelectrics, Coexistence, Dynamical Symmetry.

PACS numbers:74.20.-z,77.80.-e,64.90.+b,77.90.+k 


\section{I.Introduction, and Background.}

This paper concerns an investigation of coexistence of Superconductivity (SC) and lattice Ferroelectricity (FE) based on a model which expresses the Dynamical Symmetry underlying the physics. The model combines the dynamical symmetry of the SC and FE sub-systems into that for the composite system.

In Section II we review the algebra of the "s-wave" pairing BCS model of a superconductor . It is well known that a dynamical symmetry $s u(2)$ algebra can be identified based on time-reversed electron pair operators . The mean-field reduced Hamiltonian will be an element in this algebra. By rotating the Hamiltonian in the space of the generators to a "diagonal " form, the energies, the eigenfunctions and then the expectation value of the $\mathrm{SC}$ order parameter in the ground coherent state, is obtained.

In Section III we introduce a simplified algebraic model which has $h(4)$ dynamical symmetry for a displacive ferroelectric. It is a "displaced oscillator" model for the phonon soft-mode which has represening the soft transverse-optic (TO) mode. This Hamiltonian can also be transformed to "diagonal" form to give the energies,the eigenfunctions, and the FE order parameter in its ground coherent state.

In Section IV we introduce the SC-FE coupling , and we discuss interactions which will respect the gauge and inversion symmetries that will be broken. The Hamiltonian of the composite system including initially biquadratic interaction, is simplified using a "double mean field approximation ". The resulting bilinear Hamiltonian $\hat{H}_{D M F A}$ is in the direct product $s u(2) \otimes h(4)$ algebra, and appears to be the simplest way that the two sub-symmetries can be joined.

In Section $\mathrm{V}$ we show that although this Hamiltonian cannot be solved exactly, a variational solution can be found, by forming a trial eigenfunction analogous to the product coherent state. After carrying out the variational solution, the energy spectrum, and the eigenfunctions are obtained.

In Section VI we calculate the expectation values of the SC and the FE order parameters in the ground state of the coupled system. Our results for the order parameters 
show that : the presence of one non-zero order parameter (e.g. spontaneous polarization) tends to suppress the other (e.g. superconductivity) and vice-versa in the case of positive sign (repulsive) of the coupling between the two subsystems. This leads to the prediction that the superconducting critical temperature can increase with pressure if FE is quenched by pressure, and is in agreement with experiment in sodium tungsten bronze. Another prediction is that the ferroelectric polarization will increase with applied magnetic field . This is a new type of Magnetoelectric Effect. These predictions are discussed in Section VI.

In the final Section VII we discuss the results especially for the sodium tungsten bronze, and doped $\mathrm{SrTiO}_{3}$ systems and we suggest the relation of the present work to the mechanism of high temperature superconductivity in the copper-oxide systems as previously proposed by Peter and Weger [1], [2] based on the close proximity of a near-ferroelectric instability to the superconducting transition.

We believe the model presented here offers a simple "generic" way to treat the two broken symmetries relevant to the problem: for SC, broken gauge symmetry ( the basic pair operators do not conserve number of electrons), for FE broken inversion symmetry (spontaneous TO phonon displacement is not $i$-invariant). In passing we will show later that, if certain couplings vanish, our model can reduce to other, well-studied models: the Jaynes-Cummings Model, and the Spin-Phonon model. Our model is more general than either of these two, and truncating our model to obtain either of them would result in losing relevant physics for our case.

Apart from interest in new models for competing phases, there are several reasons for work on this problem at this time. As far back as 1964, Matthias and coworkers [3] reported superconductivity with $T_{c}$ below $1^{\circ} \mathrm{K}$ in a sodium tungsten bronze $\mathrm{Na}_{x} W \mathrm{O}_{3}$ with $0.1 \leq x \leq 1$. These authors remarked, the host crystals are isomorphic (at $x=1$ ) to barium titanate,so that it is "probable that they are also Ferroelectric,in the sense of developing a polar axis, similar again to to $\mathrm{BaTiO}_{3}$ and $\mathrm{WO}_{3}$ "' Matthias $(1949,1967)$ and Matthias and Wood (1951) [3] had confirmed the polar state for the sodium tungsten bronzes which they studied. Subsequent work of S.C.Abrahams et. al. [4], and others, has also reported 
on the structural phase transitions and the development of a polar axis in many of these compounds. The doped tungsten bronzes have also been studied as examples of the Mott and metal-insulator transitions [5]. In undoped $W_{3}$, five phase transitions have been identified: at 40, 65, 130, 220, and $260{ }^{\circ} \mathrm{K}$ [6], [7], and most recently such transitions were studied by by Aird et.al. [8]. Several recent reports of high temperature superconductivity $T_{c} \sim 90^{\circ} \mathrm{K}$ in a sodium tungsten bronze $\mathrm{Na}_{0.05} \mathrm{WO}_{3}$ system [9] added considerable new stimulous for this work, since we can suppose that the system is in a polar state at the superconducting temperature, which allows ferroelectricity.

It is worth recalling, too, that $\mathrm{SC}-\mathrm{FE}$ coexistence, and competition played a role in motivating the work of Bednorz and Muller [10] on the high temperature cuprate superconductors. Even earlier, work on the "old" superconductors of the $\beta-W$ structure like $V_{3} \mathrm{Si}, \mathrm{Nb}_{3} \mathrm{Sn}$,etc., where $T_{c} \sim 23^{\circ} \mathrm{K}$, and a martensitic phase transition occurs in the same temperature range , gave rise to investigations on the possibility of a "ferroelectric metal" or a "polar metal", and thus to the study of SC-FE coexistence [11], [12], [13], [14], [15].

A number of theoretical papers have already discussed microscopic models for the effect of lattice instability on superconductivity in the sodium tungsten bronze systems [16], [17], [18]. These papers have illuminated many aspects of the interplay between the structural deformations, such as rotation of underlying octahedaral units, and coupling with electron pairs. The present work looks at the same problem of coexistence of SC and FE from a dynamical algebra, or "Spectrum Generating Algebra [SGA]", point of view , which complements these detailed models .

Study of SC-FE coexistence problems can be relevant to recent work by Weger and collaborators [1], [2], on the mechanism of high temperature superconductivity in the cuprates. In that work the presence of a nearby FE instability close to the SC transition is related to the anomalously large ionic dielectric coefficient in the cuprates, which reduces the electron-electron repulsion and can then lead to an enhanced net electron-electron attraction, producing higher $T_{c}$.

The present work also relates to earlier dynamical symmetry investigations by Birman 
and Solomon [19], 20], [21], [22], 23], [24], [25], 26], [27] for systems with multicritical behavior involving superconductivity and charge and spin density waves. Based on the mean-field models which had earlier been used to investigate superonductor-charge density wave, and superconductor- antiferromagnetic coexistence, these papers were the first to introduce models with $S 0(6)-S 0(5)$ symmetry for Superconductor plus Charge Density Waves (SC-CDW), and Superconductor plus Antiferromagnetism (SC-AFM), as well as presenting and analysing a general $S U(8)$ "Grand Unified Theory (GUT)" model unifying singlet and triplet Superconductivity, and Charge and Spin Density Wave cooperative effects [26]. Recently, $\mathrm{SO}(5)$ and $\mathrm{SU}(4)$, models for multicritical superconductor-antiferromagnetic behavior in the high temperature superconductors have also been studied by Zhang, Demler, Guidry, and others [28], 29]. 


\section{The $s u(2)$ Pseudospin Model For a Superconductor.}

The BCS theory for superconductors can be conveniently epitomized at a mean-field level by introducing the pseudo-spin $s u(2)$ algebra of the fermion pair operators [30], 31], [32], [33]. Since this is well known, here we briefly summarize the material needed for later reference. Some more notational and other details are given in Appendix 3.

In the dynamical symmetry $s u(2)$ model for a superconductor we take the Hamiltonian in a reduced mean field approximation as

$$
\hat{H}_{S C}=\sum_{k} \hat{h}_{k}
$$

with the hamiltonian at sector $\mathrm{k}$ given as

$$
\hat{h}_{k}=-2 \epsilon_{k} \hat{j}_{3 k}+2 \Delta_{k} \hat{j}_{2 k}+2 \epsilon_{k}
$$

Here $\epsilon_{k}$ is the single electron energy, with $\epsilon_{k}=\epsilon_{k \uparrow}=\epsilon_{-k \downarrow}$, and $\Delta_{k}$ is the pairing ("gap") energy . The dynamical symmetry, or spectrum generating algebra (SGA), for each $\hat{h}_{k}$ is $s u(2)_{k}$, so the SGA of the entire Hamiltonian is $\bigotimes_{k} s u(2)_{k}$. When it causes no confusion we drop the index $k$.

The $s u(2)$ pseudo-spin operators $\hat{j}_{p k}$ obey

$$
\left[\hat{j}_{p k}, \hat{j}_{q k}\right]_{-}=i \epsilon_{p q r} \hat{j}_{r k}, \text { where }(p, q, r)=(1,2,3)
$$

and each $\hat{j}_{p k}$ is a bilinear in the fermion operators ( see Appendix 3). When all $\Delta_{k}=0$, each $\hat{h}_{k}$ is the hamiltonian for free electrons and the total wave function in the ground state is simply the product of individual creation operators acting on the vacuum state for that $k$. The filled Fermi Sea is the many-electron ground state, or the "disordered" state, denoted $|0\rangle=\prod_{k} \hat{a}_{\dagger_{k} \uparrow} \hat{a} \dagger_{-k \downarrow}|0\rangle_{k}$. For $\Delta_{k} \neq 0$ the ground state is obtained at each $\mathrm{k}$, by rotating $\hat{h}_{k}$

about the $\hat{j}_{1 k}$ axis by the angle $\theta_{1 k}=\tan ^{-1}\left(\Delta_{k} / \epsilon_{k}\right)$, so that the transformed hamiltonian will be parallel to the $\hat{j}_{3 k}$ axis.

The rotation operator is given by

$$
\hat{U}_{1 k} \equiv \exp \left(i \theta_{1 k} \hat{j}_{1 k}\right)
$$


so that

$$
\hat{h}_{k}^{\prime}=\hat{U}_{1} \hat{h}_{k} \hat{U}_{1}^{-1}=E_{k} \hat{j}_{3 k}
$$

Now $\hat{h}_{k}^{\prime}$ is directed along $\hat{j}_{3 k}$ and its eigenvalue is

$$
E_{k}=\sqrt{\Delta_{k}^{2}+\epsilon_{k}^{2}}
$$

The key ingredient needed in order to discuss a phase transition is the order operator $\hat{\eta}_{S C}$ for superconductivity. A natural choice is the real part of the pair operator, i.e. $\hat{\eta}_{S C}=$ $\hat{j}_{2 k}$. Then, the order parameter in a state is the expectation value of that order operator in that state. We single out two states : the disordered state $\left|\Psi_{\text {dis }}\right\rangle=|0\rangle$, and the ordered state $\left|\Psi_{\text {ord }}\right\rangle$. Using the identifications given above for each of these states, depending on whether $\Delta_{k}=0$, or $\neq 0$, we have $\eta_{S C}=0$,or $\eta_{S C} \neq 0$ respectively. We can go further. When $\Delta_{k} \neq 0$, the ordered state is $\left|\Psi_{\text {ord }}\right\rangle=\prod_{k}\left|\psi_{\text {ord,k }}\right\rangle=\prod_{k} \hat{U}_{1 k}^{-1}|j m\rangle$ where $|j m\rangle$ is the ususal eigenstate of $s u(2)$ (for details see Appendix 3). Then, in the ordered state we have

$$
\begin{aligned}
\eta_{S C} & \equiv\left\langle\psi_{k}\left|\hat{j}_{2 k}\right| \psi_{k}\right\rangle \\
& =\left\langle j m\left|\hat{U}_{1} \hat{j}_{2 k} \hat{U}_{1}^{-1}\right| j m\right\rangle \\
& =\left\langle j m\left|\left(\hat{j}_{2 k} \cos \theta_{1}+\hat{j}_{3 k} \sin \theta_{1}\right)\right| j m\right\rangle \\
& =m \sin \theta_{1}
\end{aligned}
$$

or

$$
\eta_{S C}=m \Delta_{k} / \sqrt{\Delta_{k}^{2}+\epsilon_{k}^{2}}
$$

The lowest (ground state) energy occurs when $m=-1 / 2$, and the order parameter $\left|\eta_{S C}\right| \neq 0$ in this state.

The states $\left|\psi_{k}\right\rangle=\hat{U}_{1}^{-1}|j m\rangle$, and in particular the ground state $\left|\psi_{k G}\right\rangle$ are $s u(2)$ coherent states in accord with the usual definitions [34], [35], [36], [37]. Globally the symmetry of the total hamiltonian $\hat{H}$, which is $\otimes_{k} s u(2)_{k}$, gives a total wave function of the ground state which is the global coherent state 


$$
\left|\Psi_{B C S}\right\rangle=\prod_{k}\left|\psi_{k G}\right\rangle
$$

The steps used in this Section will be used again below. Namely, we: a) identify the dynamical symmetry of the hamiltonian chosen; b) diagonalize the hamiltonian ,thus obtaining the ground and excited state eigenfunctions (coherent states) and energies ; c) evaluate the expectation of the relevant order operator in the appropriate state to give the order parameter. 


\section{III.Algebra h(4) for a Displacive Ferroelectric Soft Mode.}

Ferroelectricity in perovskite-like systems is due to a "soft phonon" transverse optic (TO) lattice mode resulting from ionic displacements that break inversion symmetry [38], [39], [40], [41]. When these displacements are "frozen" by higher order anharmonic terms stabilizing a distorted structure, a macroscopic FE polarization $\vec{P}$ arises; the magnitude $|\vec{P}|$ is proportional to the expectation value of the frozen soft mode amplitude $\left\langle\overrightarrow{\hat{Q}}_{0}\right\rangle$. Here $\overrightarrow{\hat{Q}}_{0}$ is the operator of the normal coordinate displacement for the soft mode [42]. In terms of the harmonic oscillator boson operators $\hat{B}_{0}, \hat{B}_{0}^{\dagger}$ of the soft mode, $\overrightarrow{\hat{Q}}_{0} \sim\left(\hat{B}_{0}^{\dagger}+\hat{B}_{0}\right)$. We will assume that only a small region in the $\vec{k}$-space softens, and we can take that to be near $\vec{k}=0$. Thus we consider a homogeneous ferroelectric material, and we neglect FE density waves or stripes which may occur if $k \neq 0$. The soft mode will be represented by a harmonic oscillator.

In the presence of spontaneous polarization $\vec{P}$, a macroscopic self-electric field $\vec{E}$ arises, giving an energy proportional to $\vec{E} \cdot \vec{P}$ [43]. The field $\vec{E}$ is the internal transverse electric field generated by the transverse polarization. The origin of the latter is the frozen TO mode. Translating this self-term into the language of our problem we shall write this energy term as $\gamma_{1} \mathcal{E}\left(\hat{B}_{0}^{\dagger}+\hat{B}_{0}\right)$, where $\gamma_{1}$ is a coupling constant we shall take as positive.

We then take as our model for the ferroelectric sector of the hamiltonian the sum of the harmonic oscillator term for the soft mode, plus the energy due to the self-field coupled to the polarization ; higher order anharmonic terms are omitted. In second quantized form this Hamiltonian is :

$$
\hat{H}_{F E}=\omega_{T 0}\left(\hat{B}_{0}^{\dagger} \hat{B}_{0}+1 / 2\right)+\gamma_{1} \mathcal{E}\left(\hat{B}_{0}^{\dagger}+\hat{B}_{0}\right) .
$$

The frequency of the soft TO mode is $\omega_{T 0}$, we take $\gamma_{1}$ as a positive constant, and $\mathcal{E}$ is the

magnitude of $\vec{E}$. We immediately recognize $\hat{H}_{F E}$ as a" displaced oscillator" hamiltonian for the soft mode, including coupling to the self-macroscopic field.

Before proceeding, note that the theory of ferroelectricity for soft mode perovskite-like systems has a long history. Microscopic models including anharmonic terms in the Hamil- 
tonian followed the original work of Cochran (1959) [38] and Anderson [39] who proposed the "soft mode" model. The models were carefully analysed by Cochran [44], Cowley [40], Cowley and Bruce [45], and others. Soon after experimental work on the doped tungsten bronzes was reported, several detailed theoretical papers appeared giving various microscopic models for the interplay between $\mathrm{SC}$ and FE, and also related to the Metal-Insulator transition in these materials [5], [0], [46], [16], [17], [18], and active study continues to the present 47.

Now we briefly comment about the soft mode. In the context of a traditional softmode displacive ferroelectric such as a perovskite like $\mathrm{BaTiO}_{3}$ or a tungsten bronze like $A_{x} W_{3}$ the soft mode can exhibit a typical temperature dependence such as:

$$
\omega_{T 0}^{2}=\Omega\left|\left(T-T_{c}\right)\right|
$$

with $\Omega$ a constant, and $T_{c}$ the FE transition temperature. On the other hand, in their very recent investigations on the mechanism of High Temperature Superconductivity in the cuprates, Weger and collaborators [1], [2], have examined quantitatively how the electronphonon and electron-electron interactions are affected by the medium itself having a high lattice-induced dielectric coefficient. For example, values of $\epsilon(\omega) \approx 50$ or more for $\omega=$ $10 m e v$, in $\mathrm{La}_{2-x} \mathrm{Sr}_{x} \mathrm{CuO}_{4}$ and YBCO were recently measured [48, [49]. As noted by these authors, the relevant soft-mode transverse optic (TO) phonon is governed by the LyddaneSachs-Teller (LST) relation [42] which for a single pair of LO and TO modes is:

$$
\epsilon(\omega)=\epsilon(\infty)\left(\omega^{2}-\omega_{L O}^{2}\right) /\left(\omega^{2}-\omega_{T O}^{2}\right)
$$

Here $\epsilon(\infty)$ is the high frequency dielectric coefficient, and $\omega_{L O, T O}$ are the characteristic phonon frequencies. This single mode expression works well for the c-axis component of the modes in both $\mathrm{La}_{2-x} \mathrm{Sr}_{x} \mathrm{CuO}_{4}$ and YBCO . In our work we will not use the explicit expression given in equation (14), but we shall make contact with the idea that the dielectric coefficient at low frequencies is very large, which is related to the "near ferroelectric instability".

We return to the Hamiltonian for the ferroelectric sector, $\hat{H}_{F E}$ It is well known 37, 
[50], [51, that the "displaced oscillator hamiltonian" can be transformed by the unitary operator

$$
\hat{U}_{2}=\exp \left[\xi_{0}\left(\hat{B}_{0}^{\dagger}-\hat{B}_{0}\right)\right]
$$

which displaces the oscillator Bose operators as

$$
\hat{U}_{2} \hat{B}_{0}^{\dagger} \hat{U}_{2}^{-1}=\hat{B}_{0}^{\dagger}+\xi_{0}
$$

We take the simplest case with $\xi_{0}$ real, and by choosing $\xi_{0}=\left(-\gamma_{1} / \omega_{T 0}\right)$, we obtain the transformed hamiltonian as

$$
\begin{aligned}
\hat{H}_{F E}^{\prime} & =\hat{U}_{2} \hat{H}_{F E} \hat{U}_{2}^{-1} \\
& =\left[\omega_{T 0}\left(\hat{B}_{0}^{\dagger} \hat{B}_{0}+1 / 2\right)-\left(\gamma_{1} \mathcal{E}\right)^{2} / \omega_{T 0}\right] .
\end{aligned}
$$

This transformed oscillator hamiltonian $\hat{H}_{F E}^{\prime}$ is shifted to a new minimum, but retains the same excitation frequency $\omega_{T 0}$ as the original oscillator.

We seek the eigenstates $|\Phi\rangle$ of the original hamiltonian

$$
\hat{H}_{F E}|\Phi\rangle=W|\Phi\rangle
$$

Transforming this equation by the operator $\hat{U}_{2}$ and continuing as in Section II, we find the eigenvalues and eigenfunctions as

$$
W_{n}=(n+1 / 2)-\left(\gamma_{1} \mathcal{E}\right)^{2} / \omega_{T 0}
$$

and

$$
|\Phi\rangle=\hat{U}_{2}^{-1}|n\rangle=\exp -\left[\xi_{0}\left(\hat{B}_{0}^{\dagger}-\hat{B}_{0}\right)\right]|n\rangle
$$

where $|n\rangle$ is a number eigenstate of the phonon number operator $\hat{N}_{B} \equiv \hat{B}_{0}^{\dagger} \hat{B}_{0}$. The state $|\Phi\rangle$ is a Glauber coherent state for the FE oscillator [37, [51].

A natural choice of the order operator for the FE polarization is the coordinate operator $\hat{Q}_{0}$, or $\left(\hat{B}_{0}^{\dagger}+\hat{B}_{0}\right)$. Thus $\hat{\eta}_{F E} \sim \hat{Q}_{0} \sim\left(\hat{B}_{0}^{\dagger}+\hat{B}_{0}\right)$. Clearly, in the state $|n\rangle$ i.e. , 
$\left|\Psi_{d i s}\right\rangle$, of the free phonon the expectation value of this order operator is zero: $\left\langle n\left|\hat{Q}_{0}\right| n\right\rangle=$ 0 , meaning no spontaneous polarization . But in the Glauber coherent state $|\Phi\rangle$, which is $\left|\Psi_{\text {ord }}\right\rangle$ we have a non-zero value of the order parameter as the following argument shows:

$$
\eta_{F E}=\left\langle\Phi\left|\hat{Q}_{0}\right| \Phi\right\rangle=\left\langle n\left|\hat{U}_{2}^{-1}\left(\hat{B}_{0}^{\dagger}+\hat{B}_{0}\right) \hat{U}_{2}\right| n\right\rangle=2 \xi_{0}=-2\left(\gamma_{1} \mathcal{E}\right) / \omega_{T 0} .
$$

If, further, we identify the magnitude of the order parameter with the macroscopic polarization we have

$$
\left|\eta_{F E}\right|=|\vec{P}|=\left(2 \gamma_{1} \mathcal{E}\right) / \omega_{T 0} .
$$

Although the field $\mathbf{E}$ is a self-field due to the spontaneous polarization, we may treat this expression as defining a macroscopic dielectric susceptibility, given as $\chi=(|\vec{P}| / \mathcal{E})=$ $2\left(\gamma_{1} / \omega_{T 0}\right)$. For a soft mode with $\omega_{T 0} \rightarrow 0$ (for example as $T \rightarrow T_{c}$ ) the susceptibility $\chi$ will become very large. This interpretation agrees with what one would expect of a ferroelectric, or "near-ferroelectric" transition. Higher order terms will stabilize the system and prevent actual divergence.

While the soft-mode displaced oscillator Hamiltonian is a very simplified version of the true state of affairs, this hamiltonian $\hat{H}_{F E}$ captures the physics of the FE sector for our purposes in this paper. Namely, this hamiltonian exhibits the FE displacement of the oscillator, which breaks a pre-existing inversion symmetry, and gives the enhanced dielectric susceptibility, in a simple algebraic $h(4)$ setting. To include higher order anharmonic or coupled terms could make the model more realistic, but would depart from our algebraic framework.

Just as we do not expect the $s u(2)$ SC model to be a microscopic model which can give all the features of superconductivity, so the $h(4) \mathrm{FE}$ model does not claim to be a microscopic model incorporating interactions needed to explain all properties of ferroelectric media. 


\section{Interaction Terms; Double Mean Field Approximation.}

\section{a) Interaction Terms}

To procede we need the total Hamiltonian which we take as

$$
\hat{H}=\hat{H}_{S C}+\hat{H}_{F E}+\hat{H}_{I N T}
$$

We will take $\hat{H}_{S C}$ and $\hat{H}_{F E}$ as before, and now we turn to $\hat{H}_{I N T}$. For the Hamiltonian to be translation invariant [52 the interaction term must match the wave vectors of the soft-mode phonon and the Cooper pairs. Since the center of mass momentum of the pairs vanishes, we will directly couple the $k=0$ lattice soft mode and the electron pairs.

In order to find the form of $\hat{H}_{I N T}$ in our algebraic framework, we need to use the basic operators in the SC and FE sectors, and combine them in an invariant fashion. It is natural to be guided by general prescriptions used in the Landau approach [53], and especially in the Ginzburg-Landau theory for competing order parameters. Here, for competing ferroelectricty and superconductivity, the order parameters are the spontaneous FE polarization $|\vec{P}|$ and the superconducting gap $\Delta$ respectively. It is required, in the G-L theory, that every term in the Free Energy shall be a scalar invariant under the relevant symmetry group, which in the present work is the direct product of the configuration space symmetry group of the crystalline medium, and the gauge group of the unbroken many-electron sector. We take the TO phonon in a prototype paraelectric crystal coupled to an "s-wave" SC complex gap parameter. For example take the prototype systems to be $W 0_{3}$ or perovskite-like, with cubic symmetry in the paraelectric and non-superconducting phase. The TO phonon will be split off from a $\Gamma\left(15^{-}\right)$or other three-dimensional representation [52]. In the homogeneous approximation where the order parameters are uniform, the Free Energy density of the system will be of the form

$$
\delta F=a|\vec{P}|^{2}+(b / 4)|\vec{P}|^{4}+\alpha|\Delta|^{2}+\beta / 4|\Delta|^{4}+\kappa / 2|\Delta|^{2}|\vec{P}|^{2}
$$

In such a G-L theory, the lowest order, "generic" coupling between superconducting $\Delta$ and the ferroelectric $\vec{P}$ will be a biquadratic term of the form proportional to $\left(|\Delta|^{2} \vec{P}^{2}\right)$, 
i.e. the last term in $\delta F$ above. This is the lowest order term which satisfies gauge and parity symmetry requirements. A similar G-L theory was analysed by Liu and Fisher [54], Imry [55], Yurkevich, Rolov and Stanley [56], and is discussed in texts such as that by Vonsovsky, Izyumov, and Kurmaev [57]. A rich phase diagram can result, depending on the relative magnitudes and signs of the coefficients of quadratic, quartic, and biquadratic terms .A case of particular interest to us, will occur for "antagonistic" order parameters for which $\kappa$ is positive. It is then possible to have four distinct stable regions in the phase diagram , including one for which there is coexistence of non-zero $\Delta$ and non zero $P$. Although our Hamiltonian will not contain terms of the fourth degree like $|P|^{4}$ or $|\Delta|^{4}$, we will carry over the positive sign of the coupling constant denoted $\gamma_{2}$ below, corresponding to $\kappa$.

We need to translate this coupling term into the operators of our model. Thus we we must add to the free hamiltonian $\hat{H}_{S C}+\hat{H}_{F E}$, the interaction term, in which we use the correspondences $|\Delta|^{2} \sim\left(\hat{j}_{2}\right)^{2}$, and $\vec{P}^{2} \sim \hat{Q}_{0}^{2}$ so that:

$$
\hat{H}_{I N T}=\gamma_{2}\left(\hat{j}_{2}^{2}\right)\left(\hat{Q}^{2}\right)=\gamma_{2}\left(\hat{j}_{2}^{2}\right)\left(\hat{B}_{0}^{\dagger}+\hat{B}_{0}\right)^{2} .
$$

We take $\gamma_{2}$, which corresponds to the $\kappa_{2}$, to be a positive coupling constant, as discussed above , in order to implement the "antagonism" of the two ordering fields. In principle both a microscopic theory of the basic interaction, and comparison with experiment ( see below in Section VI) will be needed in order to decide on the sign of $\gamma_{2}$ for a particular system.

It should also be noted that if the initial paraelectric phase is of lower symmetry than cubic , a term of lower degree for example, $|\Delta|^{2} P$ could occur. This would preserve gauge invariance, and use the component of $\hat{P}$ transforming like the identity representation of the paraelectric group. Another manner in which a coupling linear in $\hat{P}$ might arise, uses the fact that the soft mode displacement, and the associated ferroelectric polarization $\hat{P}$ is transverse in the "long-wave" limit [42] . So, in order to couple to $\hat{P}$ we should seek a transverse field operator associated to the superconductive sector. A natural candidate is the operator for the transverse superconducting current. Such a coupling term could have the form 


$$
\hat{H}_{I N T}^{\prime} \sim \hat{P}_{F E} \cdot \hat{J}_{S C}
$$

where the SC current operator would have the form

$$
\hat{J}_{S C}=\left(-i e^{*} \hbar / 2 m\right)\left[\psi^{*} \nabla \psi-\psi \nabla \psi^{*}\right)-\left(e *^{2} / m c\right)|\psi|^{2} \mathbf{A}
$$

where $e^{*}$ is the $\mathrm{SC}$ pair charge and $\mathbf{A}$ is the vector potential. In the London approximation $\mathbf{J}_{\mathbf{S C}} \sim n_{S C} \mathbf{A}$ where $n_{S C}$ is the superconducting electron density, and $\nabla \times \mathbf{A}=\mathbf{h}$, with $\mathbf{h}$ the local magnetic field. Such a term which we can also write proportional to $\mathbf{P}_{\mathbf{F E}} \cdot \mathbf{A}$ would be symmetry allowed in the correct geometry (lower than cubic, and with pre-existing broken $i$-symmetry). It would be linear in the transverse polarization. We could rewrite this term to bring it to the form of the operators of our model if we further take $J_{S C}$ proportional to the number of pairs, as $\Sigma \hat{b}_{k}^{\dagger} \hat{b}_{k}$ and then express these in terms of the $\hat{j}_{1,2}$ of the SC sector, while $\hat{P}$ would be given in terms of the Bose creation and annihilation operators $\hat{B}_{0}^{\dagger}$, and $\hat{B}_{0}$ respectively. However, since these terms are linear in $\mathbf{P}$ and refer to pre-existent broken inversion symmetry, we do not include them here.

The microscopic theory of the electron-soft ferroelectric mode (TO phonon) interaction was studied by Epifanov, Levanyuk and Levanyuk [58]. They point out that in the continuum approximation there is no transverse optic phonon to electron coupling because the TO mode does not induce a "macroscopic" electric field. By contrast, a longitudinal mode will give such a macroscopic field through the effective charge: $\nabla \cdot \hat{P}=-4 \pi \rho_{b}$ where $\rho_{b}$ is an effective "bound" charge. But, as pointed out in [58], using the correct lattice theory [42], there will be coupling of the band electron to the local internal electric field due to the TO displacement. Taking the effective mass approximation [59], a meaningful continuum approximation can be, and was, defined for the electron-soft TO mode interaction and is discussed in Epifanov et.al. [58].

In very recent work Fay and Weger [60] discuss the renormalization of the electronphonon vertex in media with large dielectric constant near the ferroelectric phase transition.

\section{b) Double Mean Field Approximation}


Assume now that the system is in a state $|\psi\rangle$ such that the product of the squares of the fluctuations of the operators $\hat{j}_{2}$, and $\hat{Q}$ evaluated in that state can be neglected. Thus if $\hat{A}$ and $\hat{B}$ are such operators and we write

$$
\hat{A}=[\hat{A}-\langle A\rangle]+\langle A\rangle \equiv \delta \hat{A}+\langle A\rangle
$$

and similarly for $\hat{B}$, then neglecting $\left\langle(\delta \hat{A})^{2}\right\rangle \times\left\langle(\delta \hat{B})^{2}\right\rangle$, we will have:

$$
\hat{A}^{2} \hat{B}^{2} \approx\left(2 \hat{A}\langle\hat{A}\rangle-\langle\hat{A}\rangle^{2}\right)\left(2 \hat{B}\langle\hat{B}\rangle-\left\langle\hat{B}^{2}\right\rangle\right)
$$

Applying this to the biquadratic interaction term we obtain

$$
\hat{H}_{I N T} \sim \hat{j}_{2}^{2}\left(\hat{B}_{0}^{\dagger}+\hat{B}_{0}\right)^{2} \approx 4 \Delta P \hat{j}_{2}\left(\hat{B}_{0}^{\dagger}+\hat{B}_{0}\right)-2 \Delta P^{2} \hat{j}_{2}-2 P \Delta^{2}\left(\hat{B}_{0}^{\dagger}+\hat{B}_{0}\right)+\Delta^{2} P^{2}
$$

Our double mean field approximation (DMFA) yields a bilinear effective interaction term $\hat{j}_{2} \hat{Q}$, and it renormalizes the coefficients of the SC pairing term $\hat{j}_{2}$ and of the linear self-term in $\hat{Q}$, and in addition there is an energy shift term proportional to $|\Delta|^{2} P^{2}$. In Appendix 1 we examine the validity of the DMFA using the variationally determined wave functions.

Recapitulating, we assume that initially we have the hamiltonian

$$
\hat{H}=-2 \sum_{k}\left(\hat{j}_{3 k}+2 \Delta_{k} \hat{j}_{2 k}\right)+\omega_{0}\left(\hat{N}_{B}+1 / 2\right)+\gamma_{1} \mathcal{E}\left(\hat{B}_{0}^{\dagger}+\hat{B}_{0}\right)+\sum_{k} \gamma_{2 k}\left(\hat{j}_{2 k}^{2}\right)\left(\hat{B}_{0}^{\dagger}+\hat{B}_{0}\right)^{2}
$$

The bare coefficients $\Delta$, and $\gamma_{1}$, refer to the prototype superconductor, and the prototype ferro-electric, and $\gamma_{2}$ is the initial pair-TO mode coupling coefficient. After making the double mean field approximation, and isolating a single mode $k$, we have the effective Hamiltonian at mode $k$ in the DMFA :

$$
\hat{H}_{D M F A}=-2 \epsilon \hat{j}_{3}+2 \Delta^{\prime} \hat{j}_{2}+\omega_{0}\left(\hat{N}_{B}+1 / 2\right)+\Gamma_{1}\left(\hat{B}_{0}^{\dagger}+\hat{B}_{0}\right)+\Gamma_{2} \hat{j}_{2}\left(\hat{B}_{0}^{\dagger}+\hat{B}_{0}\right)+\gamma_{2} P^{2} \Delta^{2} .
$$

The renormalized coefficients are $\Delta^{\prime}$, and $\Gamma_{1,2}$. This will be our working Hamiltonian . It includes the SC and the FE prototype systems and their coupling via the soft-mode oscillator coupled to the pseudo-spin pairing Hamiltonian. Note that , technically , the 
initial hamiltonian is in the "enveloping algebra" of $s u(2) \otimes h(4)$ because of the biquadratic terms, while $\hat{H}_{D M F A}$ is an element in the direct product algebra $s u(2) \otimes h(4)$. Extensions of our model to include various higher order terms coupling the phonon modes (anharmonicity) and pseudo-spin (pair-pair) terms will be treated elsewhere.

The values of the renormalized coefficients in $\hat{H}_{D M F A}$ are, in terms of the original coefficients:

$$
\begin{aligned}
& \Delta^{\prime} \equiv \Delta\left(1-\gamma_{2} P^{2}\right) \\
& \Gamma_{1} \equiv \gamma_{1} \mathcal{E}-2\left(\gamma_{2} P \Delta^{2}\right) \\
& \Gamma_{2} \equiv 4 \gamma_{2} .
\end{aligned}
$$

When $\gamma_{2} \rightarrow 0$, we recover the sum of two separate sectors: for SC and FE.

Our working model $\hat{H}_{D M F A}$ reduces to two other well-known models in certain limits: the "Jaynes-Cummings" model [61, and the "spin-phonon" model 62, 63]. The single mode "spin-boson" or "spin-phonon" model arises if $\Delta^{\prime}=0$, and $\Gamma_{1}=0$. Like our model, the spin-boson problem is not exactly soluble, but there has been much literature on it , including very recent work. Another related model is the single mode "Jaynes-Cummings" model for a photon (boson) coupled to a two-level "pseudo-spin" atom. We obtain the Jaynes -Cummings model by taking $\Delta^{\prime}=0$, and $\Gamma_{1}=0$, in $\hat{H}_{D M F A}$, and if we write

$$
\hat{j}_{2}=(1 / 2)\left(\hat{\sigma}_{+}+\hat{\sigma}_{-}\right) \equiv(1 / 2)\left(\hat{b}^{\dagger}+\hat{b}\right)
$$

where on the right hand side the $\hat{b}^{\dagger}$ and $\hat{b}$ refer to the electron pair operators, and, in the bilinear coupling term $\hat{j}_{2}\left(\hat{B}_{0}^{\dagger}+\hat{B}_{0}\right)$ and if we retain only the "energy-conserving" interaction , we obtain:

$$
\left(\sigma_{+} \hat{B}_{0}+\sigma_{-} \hat{B}_{0}^{\dagger}\right)
$$

This is the rotating-wave approximation (RWA) to the Jaynes-Cummings model. However, we cannot make this approximation for the SC-FE problem, that is for our $\hat{H}_{D M F A}$, as we will lose essential physics of our problem ; a similar point was made in the review of Leggett et.al. [63] on the spin-phonon problem. 


\section{The Variational "Coherent State" Eigenfunction.}

In order to obtain the ground state eigenfunction and eigenvalue of our model $\hat{H}_{D M F A}$, we will use a variational procedure based on the $s u(2) \otimes h(4)$ symmetry. Recall that when $\gamma_{2}=0$ the uncoupled Hamiltonian at mode $k$ is:

$$
\hat{H}=\hat{h}_{S C}+\hat{H}_{F E}
$$

Recall that this Hamiltonian is "diagonalized" by the product of two unitary transformations

previously denoted $\hat{U}=\hat{U}_{1} \hat{U}_{2}$ where the parameters $\theta_{1}=\tan ^{-1}(\Delta) / \epsilon$, and $\xi_{0}=\left(-\gamma_{1} / \omega_{0}\right)$ are the definite values obtained in Sections II and III . The resulting total state of the system without interaction $|\Psi\rangle=\hat{U}_{1}^{-1}|j m\rangle \hat{U}_{2}^{-1}|n\rangle$ is a coherent state which is the product of the coherent state of the pseudo-spin $\mathrm{su}(2)$ algebra for the SC sector, times the Glauber coherent state for the $\mathrm{h}(4)$ algebra for the FE sector.

For our coupled $\hat{H}_{D M F A}$ at mode $k$ we introduce an analogous trial variational coherent state (VCS) which is the product of two "coherent-like" states and is denoted $\left|\Psi_{v}\right\rangle$ :

$$
\left|\Psi_{v}\right\rangle=\hat{V}|j m\rangle|n\rangle=\hat{V}_{1}^{-1}|j m\rangle \hat{V}_{2}^{-1}|n\rangle
$$

where

$$
\hat{V}_{1} \equiv \exp \left(i \theta \hat{j}_{1}\right) ; \hat{V}_{2} \equiv \exp \left(\xi\left(\hat{B}_{0}^{\dagger}-\hat{B}_{0}\right)\right)
$$

but now, the parameters $\theta$, and $\xi$ are variational unknowns. The kets $|j m\rangle$ and $|n\rangle$ are as in Sections II and III. We now define the energy in state $\left|\Psi_{v}\right\rangle$ as the diagonal value of $\hat{H}_{D M F A}$ in the variational coherent state $\left|\Psi_{v}\right\rangle$

$$
\left\langle\Psi_{v}\left|\hat{H}_{D M F A}\right| \Psi_{v}\right\rangle \equiv E_{m, n}(\theta, \xi)
$$

and we determine $\theta$ and $\xi$ from

$$
\partial E_{m n} / \partial \theta=0 \text { and } \partial \mathrm{E}_{\mathrm{mn}} / \partial \xi=0
$$

The evaluation of $E_{m n}$ is particularly simple since the only non-zero contributions to the diagonal value are from the matrix elements of the operators $\hat{j}_{3}, \hat{N}_{B}$, and the constant. These enter as: 


$$
C_{3} \hat{j}_{3}+\omega_{0} \hat{N}_{B}+C_{0}
$$

where

$$
C_{3} \equiv\left(2 \epsilon \cos \theta+2 \Delta^{\prime} \sin \theta+2 \Gamma_{2} \xi \sin \theta\right)
$$

and

$$
C_{0} \equiv \omega_{0}\left(\xi^{2}+1 / 2\right)+2 \Gamma_{1} \xi+\Gamma_{2} \xi
$$

Hence,

$$
E_{m n}(\theta, \xi)=m C_{3}+n \omega_{0}+C_{0}
$$

and from this we have

$$
\tan \theta=-\left(\Delta^{\prime} / \epsilon\right)-\left(\xi \Gamma_{2}\right) / \epsilon
$$

and

$$
\xi=-\Gamma_{1} / \omega_{0}-m\left(\Gamma_{2} \sin \theta\right) / \omega_{0}
$$

As a check of these results we verify that if $\gamma_{2}=0$ then $\theta \rightarrow \theta_{1}$, and $\xi \rightarrow \xi_{0}$ of Sections 2 and 3 , with $\hat{H}_{I N T}=0$. Corrections to the expressions for $\theta$ and $\xi$ are bilinear or quadratic in the coupling constants $\Delta^{\prime}, \Gamma_{1}$, and $\Gamma_{2}$, and are not considered here. 


\section{Physical Consequences and Predictions.}

\section{a) Energy and Order Parameters}

There are some immediate physical consequences of the previous results. First, con-

sider the values of the $\mathrm{SC}$ and $\mathrm{FE}$ order parameters in the coherent state $\left|\Psi_{v}\right\rangle$, where the variational parameters $\theta$ and $\xi$ take on the values just determined.

For the SC order parameter we have

$$
\begin{aligned}
\eta_{S C}^{V C S} & =\left\langle\Psi_{v}\left|\hat{j}_{2}\right| \Psi_{v}\right\rangle \\
& =\left\langle j m\left|\left\langle n\left|\hat{V}_{j_{2}} \hat{V}^{-1}\right| j m\right\rangle\right| n\right\rangle \\
& =\left\langle j m\left|\left(\hat{j}_{2} \cos \theta-\hat{j}_{3} \sin \theta\right)\right| j m\right\rangle \\
& =-m \sin \theta
\end{aligned}
$$

or

$$
\eta_{S C}^{V C S}=m\left(\Delta^{\prime} / \epsilon+\xi \Gamma_{2} / \epsilon\right) /\left[1+\left(\Delta^{\prime} / \epsilon+\xi \Gamma_{2} / \epsilon\right)^{2}\right]^{1 / 2} .
$$

For the FE order parameter

$$
\begin{aligned}
\eta_{F E}^{V C S}=\left\langle\Psi_{v}\left|\left(\hat{B}_{0}^{\dagger}+\hat{B}_{0}\right)\right| \Psi_{v}\right\rangle & \\
& =\left\langle j m\left|\left\langle n\left|\hat{V}\left(\hat{B}_{0}^{\dagger}+\hat{B}_{0}\right) \hat{V}^{-1}\right| j m\right\rangle\right| n\right\rangle \\
& =\left\langle n\left|\hat{B}_{0}^{\dagger}+\hat{B}_{0}+2 \xi\right| n\right\rangle \\
& =2 \xi
\end{aligned}
$$

or

$$
\eta_{F E}^{V C S}=2\left(-\Gamma_{1} / \omega_{0}-\Gamma_{2}(1-m \sin \theta)\right) / \omega_{0}
$$

As a check we verified that in the absence of $\hat{H}_{I N T}$, both order parameters $\eta^{V C S}$ revert to their values for the uncoupled systems with $\Delta^{\prime} \rightarrow \Delta$ and $\xi \rightarrow \xi_{0}$.

Keeping terms up to second degree in $\Delta$ and $\mathrm{P}$ we find the order parameters in the coexisting phase . For the SC order parameter: 


$$
\eta_{S C}^{V C S}=\eta_{S C}\left(1-\gamma_{2} P^{2}\right)
$$

or

$$
\Delta^{V C S}=\Delta^{0}\left(1-\gamma_{2} P^{2}\right)
$$

For the FE order parameter:

$$
\eta_{F E}^{V C S}=\eta_{F E}\left(1-\gamma_{2} \Delta^{2}\right)
$$

or

$$
P^{V C S}=P^{0}\left(1-\gamma_{2}|\Delta|^{2}\right)
$$

In writing the above we identified the order parameters evaluated in the coexisting state with superscripts VCS, and we used superscipts 0 for the gap, and polarization values with $\gamma_{2}=0$, e.g. $\Delta^{0}$, and $P^{0}$ respectively, and we expressed the final results using the bare coupling parameter $\gamma_{2}$, instead of $\Gamma_{2}$.

At this point it is necessary to emphasize again that the sign of the parameter $\gamma_{2}$ is not fixed by any symmetry argument, but must be determined from some microscopic considerations and comparison to experiments. As we pointed out in Section IV above, we have taken $\gamma_{2}$ positive in order to implement the competition between the two types of order. With this sign taken, we immediately conclude that: the presence of one nonzero order parameter will tend to supress the other one. Thus our model supports the "Matthias Conjecture" (1967) [64, which we quote here: "Ferroelectricity seems to exclude Superconductivity more rigorously than Ferromagnetism seems to exclude Superconductivity". We take the conjecture in a weaker sense for both coexisting superconductivity and ferromagnetism, and for superconductivity and ferroelectricity. Namely : in both cases there is an "adversarial tendency", rather than some selection rule prohibiting coexistence. Since Matthias' statement, numerous examples of SC-FM and SC-AFM coexistence/competition have been found experimentally and studied theoretically. And, as pointed out in the Introduction, examples of the SC-FE coexistence are known in perovskite-type systems, and in 
alkalie tungsten bronze doped systems. Older work on the $\beta-W$ systems has considered superconductivity in "polar metals"; some examples are in [12], [13], [14], [15], [67], [65].

Returning to our results, in the pseudo-spin model of pure superconductivity, the excitation energy at each $\vec{k}$ is given by $\mathrm{E}=\left[\epsilon_{k}^{2}+\Delta_{k}^{2}\right]^{1 / 2}$. In our model,$\hat{H}_{D M F A}$, the coupling will renormalize the gap parameter $\Delta$ to $\Delta^{\prime}$. So as a first approximation we can estimate the renormalized SC sector excitation energy and gap order parameter as

$$
\begin{aligned}
& E^{\prime}=\left[\epsilon_{k}^{2}+\left(\Delta_{k}^{\prime}\right)^{2}\right]^{1 / 2} ; \\
& \Delta^{\prime}=\Delta\left(1-\gamma_{2} P^{2}\right) .
\end{aligned}
$$

or

$$
E^{\prime}=E-\left(\gamma_{2} \Delta^{2} P^{2}\right) / E^{2}+o\left(\Delta^{2} P^{4}\right)
$$

The excitation energy $E^{\prime}$ is smaller in the presence of $\vec{P} \neq 0$, and the "SC gap", $\Delta^{\prime}$ is also smaller than $\Delta$.

Similarly we note that the renormalized parameter $\Gamma_{1}$ causes a shift of the minimum potential energy of the FE oscillator from $-\left(\gamma_{1} \mathcal{E}^{2} / \omega_{0}\right)$ to $-\left(\gamma_{1} \mathcal{E}-2 \gamma_{2} P \Delta^{2}\right)^{2} / \omega_{0}$, or $\sim-\left(\gamma_{1} E^{2} / \omega_{0}+4 \gamma_{1} \gamma_{2} E P \Delta^{2} / \omega_{0}\right.$. Thus there is a smaller downward shift of the potential energy of the FE oscillator, and a less stable minimum in the FE sector.

Now consider the energy $E_{m n}(\theta, \xi)$ for which we have

$$
E_{m n}=m C_{3}+n \omega_{0}+C_{0} .
$$

We can identify the $\mathrm{SC}$ contribution as $m C_{3}$, with $m=( \pm 1 / 2,0)$, which includes some FE admixture. Also $(n+1 / 2)-\xi^{2} / \omega_{0}$ is the FE oscillator part, including some SC admixture. Such a separation is certainly not strict as the renormalized coupling constants $\Delta^{\prime}, \Gamma_{1}$, and $\Gamma_{2}$ involve all the interactions.

\section{b) Experimental Predictions}

Some experimental predictions follow from these results.

\section{i)Pressure Effect on $T_{c}$}


From

$$
\Delta^{V C S}=\Delta^{0}\left(1-\gamma_{2} P^{2}\right)
$$

we can use the BCS result that $3.52 k_{B} T_{c}=2 \Delta$. We identify $\Delta^{0} \sim T_{c}^{0}$ as the "gap" or transition temperature when there is no FE.

According to this result, the SC order parameter in the coexisting state, i.e. the "gap" $\Delta^{V C S}$, is decreased from $\Delta^{0}$ by non- zero $\hat{P}$. So, reducing $\mathrm{P}$ should increase the gap , and thus our prediction that: $T_{c}$ should increase as $\mathrm{P}$ decreases. In order to test this prediction, we need a means of reducing $\hat{P}$ by some applied field.

For most perovskite ferroelectrics, application of (positive) hydrostatic pressure $\pi$ will decrease $\mathrm{P}[41]$. Thus $d P / d \pi<0$, where $\pi$ is the applied hydrostatic pressure. If, further we assume that the effect of pressure on the bare gap (with no FE present) is small,i.e. $d \Delta^{0} / d \pi \sim 0$, then it is clear that for $\gamma_{2}>0$ and $d P / d \pi<0$ we would have as one testable consequence of the above result

$$
d T_{c} / d \pi>0
$$

Experimentally, the pressure dependence of $T_{c}$ in several Alkalie Tungsten Bronzes has been measured [68]. Of particular interest for us are the sodium tungsten bronze family in which we distinguished the materials used by Matthias and others, with $0.1 \leq x \leq 1$, and the newer materials of Reich et.al. with $x \sim 0.05$. For the "Matthias type "sodium tungsten bronze : $\mathrm{Na}_{0.23} \mathrm{WO}_{3}$, it was found that

$$
d T_{c} / d \pi \sim+1.7 \times 10^{-5 o} \mathrm{~K} / \mathrm{bar}
$$

It is tempting to attribute this as confirmation of our prediction above. But no direct measurement of change of polarization with pressure $\pi$ was made. We encourage the measurement of the pressure-dependent ferroelectric polarization $P(\pi)$ in these tungsten bronze systems, which will enable a test of our prediction.

We need also to recall other factors affecting the pressure dependence of the gap and $T_{c}$ of superconductors. It is well known [57 that a pure superconductor will exhibit 
pressure dependence of $T_{c}$ due to a number of factors : a) shift of the Fermi level under pressure, thus modifying the density of electron states at the Fermi level,b) change of phonon frequency under pressure, and c) effects of pressure on defects, to mention some factors. (Additonal complexity is exhibited,for example, in $V_{3} S i$, where hydrostatic pressure and uniaxial pressure in the [111] direction give positive coefficients, while uniaxial pressure in the [100] direction give a negative coefficient. 66] ). It is not simple to separate these effects, although for some specific cases theory was developed [14], [15], [66], 67]. Also it is well known that even in the very well studied class of $A B_{3} \mathrm{~A}-15$, or $\beta-W$ compounds, the sign of the pressure effect on $T_{c}$ can be either positive or negative for different materials, with "no apparent universality" 57.

For the $K$ and $R b$ tungsten bronzes the measured [Bloom et.al. [68] sign of the slope of pressure-dependence of $T_{c}$ is negative; however these materials have different crystal structures. As far as we can determine, there is no report of pressure effect on $T_{c}$ for the materials used by Reich et. al., i.e. sodium tungsten bronzes with $x \sim 0.05$.

To summarize : the available experiments on the older sodium tungsten bronze materials of "Matthias type" 68 agree with our prediction. If the effect of pressure on the "pure" superconducting gap (no FE present) $\Delta^{0}$ is small, then the experiments support the assumption that $\gamma_{2}>0$ in our model hamiltonian.

ii) Magnetic Field Enhancement of The Ferroelectric Polarization- A NonReciprocal Magneto-Electric Effect

Turn now to the FE order parameter $\eta_{F E}^{V C S}$, which is the spontaneus FE Polarization. Using:

$$
P^{V C S}=P^{0}\left(1-\gamma_{2}|\Delta|^{2}\right)
$$

where $P^{0}$ is the "bare" FE polarization in the absence of SC. For $\gamma_{2}>0, P$ is decreased by the presence of $|\Delta| \neq 0$. Hence, the FE polarization should increase as $\Delta$ is decreased. Application of magnetic field will decrease $\Delta$, ultimately to zero at $H_{c}$, the thermodynamic field. Hence we predict 


$$
d P / d H>0, H<H_{c} .
$$

We are unaware of any experiments testing the predicted increase of the ferroelectric polarization $\hat{P}$ with applied magnetic field for the sodium tungsten bronze samples of Matthias type $0.1 \leq x \leq 1.0$ used either by Matthias et.al. [1964,etc.] [3], or by Bloom et.al. [1976] [68]. Nor have we found any reports of such measurements on other SC-FE materials, such as the doped $\mathrm{SrTiO}_{3}$, or the newer sodium tungsten bronzes.

Our prediction of a change of the spontaneous polarization with applied magnetic field due to quenching of the superconductivity is a prediction of a new type of Magneto-Electric [ME] Effect, or more precisely, a Magneto-Polarization effect, since

$$
d P / d H \neq 0
$$

signifies the Magneto-Polarization efffect in the coexistant SC+FE system. The usual Magneto-Electric Effect, predicted by Landau-Lifshitz [43], and first discussed for $\mathrm{Cr}_{2} \mathrm{O}_{3}$ by Dzyaloshinsky, [69] is a property of materials which are usually ferromagnetic, or antiferromagnetic, whose total symmetry group includes composite anti-unitary operations which will be broken such as rotation-reflection (or inversion) combined with time-reversal. Our coexistent SC-FE system breaks inversion plus gauge symmetry. Adding the applied magnetic field to quench superconductivity breaks time reversal $\Theta$, and places the SC-FE in presence of an external $\hat{B}$ field in a symmetry class for the ME effect [70], [71].

Thus a material in the coexistent superconducting-ferroelectric state ,in the presence of an applied external magnetic field will exhibit broken inversion plus time reversal and broken gauge symmetries. Hence our new Magneto-Polarization Effect is allowed. If we now use one of W. Pauli's famous aphorisms : " Anything not prohibited (by symmetry) is mandatory" we can then predict the existence on symmetry grounds of this new class of Magneto-Electric, or Magneto-Polarization Effect in a Superconducting Ferroelectric. There is, however one important remark to be made. Unlike the usual ME effect, in the SC-FE case, the effect is not "reciprocal" between magnetic field and applied electric field. 
Previous work on the Magneto-Electric Effect has been reviewed authoritatively , [70], [71]. But, to our knowlege our prediction is the first of this new Magneto-Electric Effect in a Superconducting-Ferroelectric material. 


\section{VII . Discussion and Conclusions.}

The present work was motivated by our attempt to formulate the simplest algebraic model which would embody the relevant dynamical symmetries to describe the multicritical point for coupled superconductivity and ferroelectricity . In that framework it is natural to use the pseudo-spin $s u(2)$ algebra for superconductivity, since this algebra expresses the breaking of gauge symmetry embedded in the BCS theory [1957] [30], and has proven useful in other contexts. We introduced the linearly displaced oscillator for the soft-mode phonon in describing a ferroelectric via the $h(4)$ "Heisenberg Algebra" since this captures several key aspects of the displacive ferroelectric transition. Namely the shift to a new equilibrum, which breaks a pre-existing inversion symmetry and allows a spontaneous ferroelectric (and pyroelectric) moment, and the high dielectric coefficient. The simplest invariant coupling of these two order parameters which respects both the gauge and the inversion symmetries requires a biquadratic coupling which then turns to a bilinear coupling when reduced via our "Double Mean Field Approximation "[DMFA] . The Hamiltonian $\hat{H}_{D M F A}$ is an element in $s u(2) \otimes h(4)$.

The physical idea motivating the present work is that the superconducting and ferroelectric transitions are close to one another at a multicritical point. As we know from Ginzburg-Landau theory and renormalization group theories of such multicritical behavior, each transition will renormalize the other. A simple example of this is that our double mean field approximation (DMFA) renormalizes the "bare" coupling constants (see equation (35)), and leads to shifts in the two order parameters (see equations (60),(63)) from bare values. Hence the frustration, or reduction, of one order parameter will enhance the other. This is consistent with the weaker form of the "Matthias Conjecture" on the mutually antagonistic effects of superconductivity and ferroelectricity as shown earlier in this paper. Matthias was led to this statement in part by his work on the sodium tungsten bronzes. The new work by Reich et.al. on the sodium tungsten bronzes (in a different sodium composition range) reopens interest in the particular questions related to the superconducting-ferroelectric competition. We hope that our predictions in this paper and elsewhere on electrodynamics [72] 
will stimulate further experimental work on these known coexistent systems including the titanate and bronze systems.

Now we turn to possible relevance of this scenario to the high temperature cuprates. As was noted by Weger and collaborators [1], [2], conventional Eliashberg-McMillan theory of phonon-mediated interaction does not consider a multicritical point,nor in particular, the case where one phonon is a soft-mode ferroelectric phonon. There is no direct neutron scattering evidence for a soft ferroelectric phonon, however there is evidence that a close nearferroelectric lattice instability exists in cuprates, and can play a role in the superconductivity mechanism.

The measurements of ionic dielectric coefficients in several high $T_{c}$ cuprates shows exceptionally high values of the dielectric coefficients. Thus in $\mathrm{YBCO}$ and $\mathrm{LaSrCuO}$ values of $\epsilon \sim 40-50$ were measured below dispersion frequencies of 19 mev and $27 \mathrm{mev}$, respectively. These values are reminiscent of the well-known near-ferroelectricity in the perovskites. [Recall that enhancement of $\mathrm{SC}$ by nearby FE was predicted for doped $\mathrm{SrTiO}_{3}$ in 1964 by M.L.Cohen [73], and was investigated thoroughly by A.Baratoff et.al.(1981) [74]. By itself doped $\mathrm{SrTiO}_{3}$ is not expected to be a superconductor, but rather a nearly ferroelectric semiconductor. However,the electron-phonon interaction is enhanced by the near-ferroelectricity, and possibly by the multivalley conduction band, and as a result doped $\mathrm{SrTi}_{3}$ is a superconductor with $T_{c} \sim 1.5^{\circ} \mathrm{K}$.]

In order to identify the phonons responsible for the high dielectric coefficient in the cuprates consider the dispersion of $\epsilon(\omega)$, which identifies the c-axis motion of the alkaline earth ion $(\mathrm{Ba}$ in $\mathrm{YBCO}, \mathrm{Sr}$ in $\mathrm{LaSrCuO})$ as responsible for the large values of $\epsilon$. These are not the phonons inducing the superconducting pairing, which are the planar oxygen displacements, at around $35 \mathrm{mev}$ (transverse) according to [75], and possibly near $70 \mathrm{mev}$ (longitudinal).

We can then suggest the following picture which will relate to our model: the usual electron-acoustic phonon and possibly other electron-non-soft phonons are responsible for electron-pairing [76], and their effect is present in the "pairing" coefficient $\Delta$ in $\hat{H}_{S C}$, 
which carries over to the coupled Hamiltonian $\hat{H}_{D M F A}$, which we use in our analysis. The separate electron-soft-mode or ferroelectric phonon represented by operators $\hat{B}_{0}^{\dagger}$ and $\hat{B}_{0}$, with characteristic phonon frequency $\omega_{T 0}$ then couples to the formed electron pairs, giving the term $\hat{H}_{I N T}$.

In the language of Eliashberg-McMillan theory [77], [78] the electron-phonon coupling constant $\lambda_{\infty}$ for coupling to phonon mode $\Omega_{A}$ is renormalized by proximity to a ferroelectric transition, so that the constant becomes frequency-dependent as $\lambda(\omega) \sim \epsilon^{2}(\omega) \lambda_{\infty}$ [1], and also is large at low frequencies $\omega \ll \Omega_{A}$,i.e. away from the physical sheet. This is consistent with the small phonon shifts in the superconducting state for , e.g. YBCO 779 where we can argue that $\lambda(\omega)$ is very large. Note, however, large shifts were observed in $\mathrm{BaCaHgCuO}$ 80 and in organic salts 81.

Making a short digression from the cuprates we can remark that the data on $(B E D T-T T F)_{2} C u(C N S)_{2}$ are illuminating in this context.The frequency of the phonon at 2 mev increases by almost $20 \%$ below $T_{c}$. The superconducting gap in this material is $2 \Delta=10 \mathrm{meV}$ 82]. According to the theory of Zeyher and Zwicknagl [79], the phonon frequency should be pushed down below $T_{c}$, while experimentally it increases. We suggest that the mode at 2 mev is the soft or nearly "ferroelectric" phonon, which is not the one responsible for the pairing ; the phonons responsible for the pairing being around 6-8 mev. According to the results presented in this paper, superconductivity suppresses the tendency to ferroelectricity and thus, in effect, it "hardens" the relevant phonon.

The data on $\mathrm{HgBa}_{2} \mathrm{Ca}_{3} \mathrm{Cu}_{4} \mathrm{O}_{10}$ [80 is that the mode at $30 \mathrm{meV}$ softens at $\mathrm{T}_{c}$ by about $7 \%$, and the mode at $50 \mathrm{meV}$ by about $10 \%$. Thus the direction of the effect is opposite to that in the organic superconductor. Hadjiev et.al. [80] account for the effect as being due to the enormous anisotropy (which is the cause of the d-wave pairing). We do not consider this in the present work. Also, the frequency of the phonon is considerably higher than that of the "ferroelectric" mode, which is 19 mev in YBCO.

The cited infra-Red measurements have shown [48, 49], that the ionic dielectric 
constant is dominated by the c-axis motion of the alkaline earth ion . Additional support for the existence of a large ionic dielectric coefficient associated with the motion of the $\mathrm{Sr}$ ion and its connection to the electronic properties of this material can be found from the EXAFS measurements of Pollinger et al [83] which show an anomaly in the distances of Sr to Oxygen in the $\mathrm{LaSrCuO}$ material. The large difference in distances between La-O and Sr-O supports a large polarization associated with the Sr. The interpretation is in terms of a Zhang-Rice singlet and an anti-Jahn Teller triplet which are nearly degenerate in energy. Some theoretical calculations of Anisimov -Andersen,and Kamimura, [84, 85, support the near degeneracy, which contributes to the high value of $\epsilon$.

Another way to obtain the value of dielectric coefficient is via the small difference in energies between the Zhang-Rice singlet and the Jahn-Teller triplet. We can call this $U_{\text {eff }}=U_{\text {bare }} / \epsilon$, which can serve as a definition of $\epsilon$. Now, if we assume that this $\epsilon$ is just that ionic dielectric coefficient actually measured in the IR experiments, then $U_{\text {eff }}(\omega)$ will be frequency dependent. Further, the small $U_{\text {eff }}$ is associated with the lower edge of the "mid-IR" band, and it is described by a Jahn-Teller picture, as analysed by Moskvin et.al.(1998) [86]. Added support for the identication of a small $U_{\text {eff }}$ can come from the report that there is a softening of a longitudinal mode at a wave-vector near the zone boundary. Since this can indicate a tendency for charge segregation between the coppers it is consistent with a small $U_{\text {eff }}$.

Returning to the sodium tungsten bronze systems there is a clear case for the applicability of this model. Pure $W_{3}$ is ferroelectric, with large $\epsilon$, so the reported High $T_{c}$ [9] gives a picture of a material which is a high $T_{c}$ system in the presence of ferroelectricity but without copper which was previously anticipated to be ubiquitously associated with high temperature superconductivity. In the new bronzes now under study [9], the superconductivity seems to be restricted to portions of the physical surface of the material ; this could be either an inherent effect, or possibly due to a preferential sodium concentration on the surface. The recent STM data of Levi, Millo et.al. [9] indicates a superconducting gap which is sharp (as for s-wave pairing) but much smaller than in YBCO, suggesting weak or 
intermediate coupling strength. Thus if the pairing is due to phonons, the frequency of the pairing phonon must be much higher-about $70 \mathrm{meV}$, as for example due to longitudinal oxygen displacements. Ferroelectricity here would involve displacements of the tungsten atoms, again consistent with two types of phonons playing different roles.

In short, the cuprates may present a more complicated situation than the titanates or bronzes because the role of a latent or near-ferroelectric soft mode instability is partly masked - this is not surprising in view of the more complicated chemical composition and possible effects of magnetic excitations, as well as disorder in the cuprates. In the strontium titanate and sodium tungsten bronze cases application of the model seems straightforward, and it captures symmetry-related aspects of the coexistence-competition of the two collective effects. At a microscopic level our picture supports the view that there are two kinds of phonons playing a decisive role in these systems with SC-FE coexistence : first, the usual Frohlich coupled acoustic phonons giving the familiar BCS electron-electron pairing effects , and secondly, the, soft-mode ferroelectric phonons which yield the strongly enhanced static dielectric coefficient (e.g. via the Lyddane-Sachs-Teller related physics). As discussed elsewhere [1], [2], the latter phonons give an enhanced Thomas-Fermi screening length , which in turn changes the electron-phonon coupling and then the net effective electronelectron coupling, so that when inserted in the Eliashberg equations the new physics arises.

The dynamical symmetry model proposed here captures certain essential features of the superconductor-ferroelectric competition/coexistence. Predictions of pressure and magnetic-field effects can be tested and will enable the sign of the coupling constant $\gamma_{2}$ to be determined. For a given material the same coefficient will regulate the change in $T_{c}$ under pressure and the ferroelectric polarization under magnetic field as given in Section VI. Novel electrodynamic effects which are predicted for a nearly-ferroelectric superconductor are discussed elsewhere [72].

\section{VIII . Acknowledgements}

It is a pleasure to acknowledge helpful discussions and comments at various times from Professor Y. Avron, Professor C.W.Chu, Professor C.S.Ting, Dr. Mikhail Chernikov, 
Professor M.E.Fisher, and Professor David Schmeltzer. Also help from Ms.Nguyen Que Huong is gratefully acknowledged (J.L.B.). J.L.B. acknowledges with thanks the hospitality of Profesor Ady Mann, and Professor Michael Revzen, and the Physics Department, Technion, Haifa, Israel, where part of this work was carried out. 


\section{Appendix 1: Calculation of Variances, and the DMFA.}

In order to examine the validity of the DMFA we need to calculate variances of the operators $\hat{j}_{2}^{2}$ and $\hat{Q}^{2}=\left(\hat{B}_{0}^{\dagger}+\hat{B}_{0}\right)^{2}$. First, we estimate the corrections to the parameters $\theta$ and $\xi$ due to adding the interaction Hamiltonian $\hat{H}_{I N T}$, which is characterized by the strength $\gamma_{2}$, to the non-interacting $\hat{h}_{S C}$ and $\hat{H}_{F E}$. Going back to equation (54) and the renormalized coefficients given in equation (55), after a little algebra, and retaining terms of lowest (linear ) order in $\gamma_{2}$, we obtain:

$$
\tan \theta=\left(\tan \theta_{1}\right)\left(1-\gamma_{2} P^{2}\right)
$$

and ,

$$
\xi=\xi_{0}-4 m \gamma_{2} \sin \theta_{1}
$$

where $\sin \theta_{1}=(\Delta / \epsilon) / \sqrt{\left[1+(\Delta / \epsilon)^{2}\right]}$. Next, we note that $\hat{j}_{2}^{2}=(1 / 4)\left(2 \hat{n}_{k} \hat{n}_{-k}-2 \hat{j}_{3}\right)$ so that we will be able to easily find the expectation values directly. We then calculate

$$
\hat{V}_{1} \hat{j}_{2}^{2} \hat{V}_{1}^{-1}=2 \cos ^{2} \theta \hat{j}_{2}^{2}+(1 / 2) \sin 2 \theta \hat{j}_{2}+\sin ^{2} \theta \hat{j}_{3}^{2}
$$

with $\hat{V}_{1}=\exp \left(i \theta \hat{j}_{1}\right)$ as in equation (39). We then find

$$
\left\langle\left(\delta \hat{j}_{2}\right)^{2}\right\rangle=\left\langle\left(\hat{j}_{2}\right)^{2}\right\rangle-\left(\left\langle\hat{j}_{2}\right\rangle\right)^{2}=3 \cos ^{2} \theta
$$

and then as a figure of merit we can take:

$$
\left\langle\left(\delta \hat{j}_{2}\right)^{2}\right\rangle /\left(\left\langle\hat{j}_{2}\right\rangle\right)^{2}=\cot ^{2} \theta_{1}\left(1+(1 / 2) \gamma_{2}^{2} P^{2} \sin ^{2} \theta_{1}\right) /\left(1-\gamma_{2} P^{2}\right)
$$

Substituting the value of $\tan \theta_{1}$, this normalized variance can then be expressed in terms of the parameters of the Hamiltonian as:

$$
\left.\left\langle\left(\delta \hat{j}_{2}\right)^{2}\right\rangle\right) /\left(\left\langle\hat{j}_{2}\right\rangle\right)^{2}=12(\Delta / \epsilon)^{2}\left(\left(1+\gamma_{2} P^{2} \sin ^{2} \theta_{1}\right) /\left(1-\gamma_{2} P^{2}\right)\right)
$$

Apart from the numerical factor, the scale of the variance is set by $(\Delta / \epsilon)^{2}$ which is clearly

$\ll 1$. This will be true as well when the FE Polarization is non zero. Consequently we 
verify that the variance of the operator representing SC ( the real part of the gap operator) is negligable in the variational coherent state.

Turning to the variance of $\hat{Q}$, we need to calculate it using the operator $\left(\hat{B}_{0}^{\dagger}+\hat{B}_{0}\right)^{2}$, but this is equal to $\left(\hat{B}_{0}^{\dagger}\right)^{2}+\left(\hat{B}_{0}^{2}\right)+2 \hat{N}_{B}+1$. Then the matrix elements are easily evaluated:

$$
\left\langle n\left|\hat{V}_{2} \hat{Q}^{2} \hat{V}_{2}^{-1}\right| n\right\rangle=2 n+4(\xi)^{2}+1
$$

and

$$
\left\langle n\left|\hat{V}_{2} \hat{Q} \hat{V}_{2}^{-1}\right| n\right\rangle=2 \xi
$$

The normalized variance is then:

$$
\left(\left\langle\hat{Q}^{2}\right\rangle-(\langle\hat{Q}\rangle)^{2}\right) /(\langle\hat{Q}\rangle)^{2}=\left((2 n+1) / 4(\xi)^{2}\right)
$$

Since $\xi \sim P / 2+o\left(\gamma_{2}\right)$, and $\mathrm{P}$ will be large in the FE state, we verify that $\left(\left\langle\delta \hat{Q}^{2}\right\rangle\right) \ll 1$ in the variational coherent state $\left|\Psi_{v}\right\rangle$.

We may then conclude that the DMFA, in the variational coherent state approximation with wave function $\left|\Psi_{v}\right\rangle$ is a self-consistent approximation. 


\section{Appendix 2: Correlators}

Using our model we can examine the thermal average of two-time correlators of the basic operators of the model: the FE phonon displacement operator $\hat{Q}$, and the real part of the pair operator $\hat{j}_{2}$. The thermal average of the two time correlator of an operator $\hat{O}_{\alpha}$ is:

$$
\left\langle\left\langle\hat{O}_{\alpha}(t) \hat{O}_{\alpha}(0)\right\rangle\right\rangle=(1 / Z) \operatorname{Tr}(\exp -(\beta \hat{H})) \exp (i \hat{H} t) \hat{O}_{\alpha} \exp (-i \hat{H} t) O_{\alpha}
$$

The partition function is $Z=\operatorname{Tr}^{-\beta \hat{H}}$ with $\beta=\left(k_{B} T\right)^{-1}$. Cross correlators can be similarly defined, by replacing one of the $\alpha$ subscipts by a different index referring to another of the operators.

Calculation of these correlators is not possible if we use the full Hamiltonian, or even if we use $\hat{H}_{D M F A}$. However if we denote the transformed $\hat{H}_{D M F A}$ by $\hat{H}^{\prime}$

$$
\hat{H}^{\prime}=\hat{V} \hat{H}_{D M F A} \hat{V}^{-1}
$$

and evaluate $\hat{H}^{\prime}$ at $\theta=\theta_{V C S}$ and $\xi=\xi_{V C S}$, we may then take as a good approximation

$$
\hat{H}^{\prime} \sim C_{3}+\omega_{0} \hat{N}_{B}+C_{0}
$$

Making this Ansatz will permit us to easily evaluate the operators in Heisenberg picture, and then evaluate the traces. This is consistent with retaining the dominant leading order in the coupling parameters $\Delta, \gamma_{1}$, and $\gamma_{2}$.

We first work out the displacement-displacement correlator. Using invariance of the trace we can then write

$$
\langle\langle\hat{Q}(t) \hat{Q}(0)\rangle\rangle=(1 / Z) \operatorname{Tr}\left[\hat{V} e^{-\beta \hat{H}} \hat{V}^{-1} \hat{V} e^{i \hat{H} t} \hat{V}^{-1} \hat{V} \hat{Q} \hat{V}^{-1} \hat{V} e^{-i \hat{H} t} \hat{V}^{-1} \hat{V} \hat{Q} \hat{V}^{-1}\right]
$$

Using the properties of the operators $\hat{Q}$ and $\hat{H}$ transformed under $\hat{V}$ we obtain an intermediate result

$$
\langle\langle\hat{Q}(t) \hat{Q}(0)\rangle\rangle=(1 / Z) \operatorname{Tr}\left[e^{i \beta \omega_{0} \hat{N}_{B}}\left(e^{i \omega_{0} t} \hat{B}_{0}^{\dagger}+e^{-i \omega_{0} t} \hat{B}_{0}+2 \xi\right)+2 \xi e^{-\beta \omega_{0} \hat{N}_{B}}(\hat{Q}+2 \xi)\right] .
$$

As only the lattice oscillator Bose operators have survived we now carry out the diagonal summation over the harmonic oscillator quantum number $n$. We then find 


$$
Z(\beta)=e^{\beta \omega_{0} / 2} \times\left[2 \sinh \left(\beta \omega_{0}\right)\right]^{-1}
$$

and finally

$$
\langle\langle\hat{Q}(t) \hat{Q}(0)\rangle\rangle=4 \xi^{2}+e^{-i \omega_{0} t}+2 Z(\beta) \cos \omega_{0} t
$$

The same correlator can be evaluated for a Hamiltonian $\hat{H}_{F E}^{0}=\omega_{0}\left(\hat{N}_{B}+1 / 2\right)$, without the linear displaced term ; it gives the same result as above without the term $4 \xi^{2}$. The value of $\xi$ to

be taken here is $\xi_{V C S}$ from the solution of the variational problem.

In exactly the same way, but now doing the trace over the 4 electronic states with $m=0,0, \pm 1 / 2$ we obtain:

$$
\left\langle\left\langle\hat{j}_{2}(t) \hat{j}_{2}(0)\right\rangle\right\rangle=\left(\sin ^{2} \theta_{V C S} \times \cosh \beta C_{3}\right)\left[4\left(1+\cosh \beta C_{3}\right]^{-1}\right.
$$




\section{Appendix 3-Some $s u(2)$ results.}

In this Appendix we record some known results, which will be useful in various

calculations throughout the paper.If $\hat{a}_{k \uparrow}, \hat{a}_{k \uparrow}^{\dagger}$ are the electron annihilation/creation operators for wave vector $\vec{k}$, spin $(\uparrow)$, the relevent pair operators are defined by

$$
\hat{b}_{k}^{\dagger} \equiv \hat{a}_{k \uparrow}^{\dagger} \hat{a}_{-k \downarrow}^{\dagger} ; \hat{b}_{k}=\left(\hat{b}_{k}^{\dagger}\right)^{\dagger} ; \quad \hat{n}_{k} \equiv \hat{a}_{k \uparrow}^{\dagger} \hat{a}_{k \uparrow} .
$$

The $s u(2)$ pseudospin algebra at each $\vec{k}$ is generated by

$$
\begin{gathered}
\hat{j}_{1 k} \equiv(-i / 2)\left(\hat{b}_{k}^{\dagger}-\hat{b}_{k}\right), \\
\hat{j}_{2 k} \equiv(1 / 2)\left(\hat{b}_{k}^{\dagger}+\hat{b}_{k}\right), \\
\hat{j}_{3 k} \equiv(-1 / 2)\left(\hat{n}_{k}+\hat{n}_{-k}-1\right)
\end{gathered}
$$

so that

$$
\left[\hat{j}_{p k}, \hat{j}_{q k}\right]_{-}=i \epsilon_{p q r} \hat{j}_{r k}, \text { where }(p, q, r)=(1,2,3)
$$

A basic set of states for each sector $\hat{h}_{k}$ can be obtained by starting from $s u(2)$ eigenstates labelled by a pair of indices . Thus, since the eigenvalues of $\hat{n}_{ \pm k}$ are $(0,1)$ the states can be labelled by the eigenvalues $\left(n_{k}, n_{-k}\right)$. Or, we can use the $(j m)$ labels of the ket $|j m\rangle$ referring to $s u(2)$ (suppressing $k$ ), which is an eigenstate of $\hat{j}^{2}$ and of $\hat{j}_{3}$. Here

$$
\hat{j}_{3}|j m\rangle=m|j m\rangle ; \quad \hat{j}^{2}|j m\rangle=j(j+1)|j m\rangle \text {. }
$$

where we note that $\hat{j}^{2}=\left(\hat{n}_{k} \hat{n}_{-k}+\hat{j}_{3 k}+\hat{j}_{3 k}^{2}\right)$. Thus $\hat{j}^{2}$, as well as $\hat{j}_{3 k}$, depends only on the number operators for $\pm k$ and so their eigenvalues are easily computed from the allowed eigenvalues $\left(n_{k}, n_{-k}\right)$. Also the same states are obtained by applying creation operators to the vacuum state like $\hat{a}_{k}^{\dagger}|0\rangle$ etc. Enumerating these states at each $\mathrm{k}$ we have:

$$
(0,0) \sim|3 / 4,1 / 2\rangle \sim|0\rangle
$$




$$
\begin{gathered}
(0,1) \sim|0,0\rangle \sim \hat{a}_{-k \downarrow}^{\dagger}|0\rangle \\
(1,0) \sim|0,0\rangle \sim \hat{a}_{k \uparrow}^{\dagger}|0\rangle \\
(1,1) \sim|3 / 4,-1 / 2\rangle \sim \hat{a}_{k \uparrow}^{\dagger} \hat{a}_{-k \downarrow}^{\dagger}|0\rangle \sim \hat{b}_{k}^{\dagger}|0\rangle
\end{gathered}
$$

In each line, the states are presented in order, labelled by $\left(n_{k}, n_{-k}\right)$, by $|j, m\rangle$, and finally by creation operators applied to the vacuum $|0\rangle$. In constructing the eigenstates for $\hat{h}_{k}$ these basic states of the free hamiltonian will be rotated to produce the $s u(2)$-coherent states, which are eigenstates of $\hat{h_{k}}$. Recall [21] that the states $(0,0)$ and $(1,1)$ are the basis for the irreducible representation $D^{(1 / 2)}$, and $(0,1)$ and $(1,0)$ are bases for $D^{(0)}$, of $s u(2)$.(N.B. The latter two states are a time reverse pair for the corepresentation of $s u(2) \otimes \Theta)$.

The eigenstates of $\hat{j}_{3 k}$ are the basic set $|j m\rangle$ given above. To obtain the eigenstates $\left|\psi_{k}\right\rangle$ or $\left|\Psi_{\text {ord }}\right\rangle$, of the original problem

$$
\hat{h}_{k}\left|\psi_{k}\right\rangle=\lambda_{k}\left|\psi_{k}\right\rangle
$$

transform by $\hat{U}_{1}$ as follows

$$
\hat{U}_{1} \hat{h}_{k} \hat{U}_{1}^{-1} \hat{U}_{1}\left|\psi_{k}\right\rangle=\lambda_{k} \hat{U}_{1}\left|\psi_{k}\right\rangle
$$

and then we find

$$
\lambda_{k}=m E_{k}, \text { and }\left|\psi_{k}\right\rangle=\hat{U}_{1}^{-1}|j m\rangle .
$$

Now we can evaluate the order parameter in the state $\left|\psi_{k}\right\rangle=\left|\Psi_{\text {ord }}\right\rangle$ :

$$
\eta_{S C} \equiv\left\langle\psi_{k}\left|\hat{j}_{2 k}\right| \psi_{k}\right\rangle=\left\langle j m\left|\hat{U}_{1} \hat{j}_{2 k} \hat{U}_{1}^{-1}\right| j m\right\rangle=\left\langle j m\left|\left(\hat{j}_{2 k} \cos \theta_{1}+\hat{j}_{3 k} \sin \theta_{1}\right)\right| j m\right\rangle=m \sin \theta_{1}
$$

or

$$
\eta_{S C}=m \Delta_{k} / \sqrt{\Delta_{k}^{2}+\epsilon_{k}^{2}}
$$


The lowest (ground state) energy $\lambda_{G}$ occurs when $m=-1 / 2$, and the corresponding order parameter $\left|\eta_{S C}\right| \neq 0$, and $\lambda_{G}=(-1 / 2) E_{k}$. The ground eigenstate $\left|\psi_{k G}\right\rangle$ is the BCS pair state which can be written in several alternate forms. These are

$$
\begin{aligned}
\left|\psi_{k G}\right\rangle & =\exp \left(-i \theta_{1} \hat{j}_{1 k}\right)|3 / 4,-1 / 2\rangle \\
& =\exp -\left[\left(\theta_{1} / 2\right)\left(\hat{b}_{k}^{\dagger}-\hat{b}_{k}\right)\right] \hat{b}_{k}^{\dagger}|0\rangle \\
& =\hat{U}^{-1}|3 / 4,-1 / 2\rangle .
\end{aligned}
$$

or

$$
\left|\psi_{k G}\right\rangle=-\left(u_{k}+v_{k} \hat{b}_{k}^{\dagger}\right)|0\rangle
$$

with $u_{k} \equiv-\sin \left(\theta_{1} / 2\right) ; v_{k} \equiv \cos \left(\theta_{1} / 2\right)$.

In passing we note that we can recover the BCS gap equation from the above. The $\eta_{S C}$ given above is for one sector $\mathrm{k}$. If we assume that $\Delta_{k}=V \Delta$,i.e. is independent of $k$ and that the "global" order parameter is

$$
\Delta=\sum_{k} \eta_{S C(k)}
$$

we may then obtain a self-consistent equation for the total gap function as:

$$
\Delta=\sum_{k} \eta_{S C(k)}=(1 / 2) \sum_{k}\left(V \Delta_{k}\right) / \sqrt{\epsilon_{k}^{2}+\Delta_{k}^{2}}
$$

and substituting the above we have:

$$
2=\sum_{k}(V) / \sqrt{\epsilon_{k}^{2}+\Delta_{k}^{2}}=V \int \rho(\epsilon) d \epsilon / \sqrt{\epsilon^{2}+\Delta^{2}}
$$

Here,we changed variables to energy, and $\rho(\epsilon)$ is the density of states (at the Fermi level) This reproduces the familiar result

$$
(2 / V)=\int \rho(\epsilon) d \epsilon / \sqrt{\epsilon^{2}+\Delta^{2}}
$$




\section{REFERENCES}

[1] M.Peter and M.Weger, "Influence of Ionic Dielectricity on the Dynamic Superconducting Order Parameter". in "High Temperature Superconductivity", ed. S.E.Barnes et.al., Am.Inst.of Phys. (1999), p.90-103.

[2] M.Peter, M.Weger, L.P.Pitaevskii, Ann.Physik 7, 174-200 (1998); Physica C317 - 318, 252 (1999); M. Weger, J. Supercond. 10, 435 (1997).

[3] C.J.Raub, A.R.Sweedler, M.A.Jensen, S.Broadston, B.T.Matthias, Phys.Rev.Lett.13 ,746 (1964); B.T.Matthias, Phys.Rev. 76, 430 (1949); B.T.Matthias and E.A.Wood, Phys.Rev.83,1255 (1951) [on $\mathrm{FE}$ in $\left.W 0_{3}\right]$; B.T.Matthias in Ferroelectricity, ed.E.Weller,Elsevier Pub.Co.Amsterdam,p.181-2 (1967).

[4] S.C.Abrahams,et.al.,J.Chem.Phys.48,5048(1968);ibid.50,4352(1969);ibid.54,23 (1971).

[5] N.F. Mott, Philosoph.Mag. 35,111(1977); L.Friedman,J.Non Cryst. Solids 6,6(1971).

[6] I.Lefkowitz, et.al., J.Solid State Chem. 15, 24.11 (1975).

[7] D.F.Holcomb,Ann.der Physik,8,no7-9,649 (1999); A.Stashans, et.al., Int.Journ. of Quant.Chem., 63,no.3, 729 (1997); N.N.Garif'yanov et.al., Czech.Jnl. of Phys. 46, supp.pt.S2,855-6(1996); H.Ducker, et.al. Journal of Non Cryst.Solids ,205 - 207,part 1 ,32 (1996); N.N.Garif'yanov, et.al. Jnl.Expt. and Theor.Phys. 80 no 2, 301(1995).

[8] A.Aird and E.K.Salje, J.Phys.Cond.Mat.10,L377,L569 (1998).

[9] S.Reich and Y.Tsabba, Eur.Phys.Journal 9 ,1 (1999); A.Shengelaya, S.Reich, Y.Tsabba, K.A.Mueller, Eur. Phys. J. B12, 13 (1999); Y.Levi, O.Millo, A. Sharoni, Y. Tsabba, G.Lectus, S. Reich, Europhysics Letters , 51, 564 (2000).

[10] J.G.Bednorz and K.A. Muller ,Rev.Mod.Phys. 60, 685 (1988), and papers referred to therein.

[11] M.Weger and I.M. Goldberg ,in Solid State Physics 28 (1973) ed. F.Seitz, D. Turn- 
bull,and H . Ehrenreich. Also, see references in this review article.

[12] P.W.Anderson and E.Blount, Phys.Rev.Lett.14,217 (1965).

[13] J.L. Birman, Phys.Rev.Lett.17,1216 (1966).

[14] C.S.Ting and J.L.Birman, Phys.Rev.B 12,1093 (1975).

[15] R. N. Bhatt and W. Mc Millan ,Phys. Rev.B14,1007 (1976).

[16] K.L.Ngai, R.Silberglitt, Phys.Rev.B,13,1032 (1976).

[17] K.Ngai,T.Reinecke,Phys.Rev.Lett.,38, $\quad$ 74(1977); $\quad$ Phys.Rev.B,16,1077(1977); J.Phys.F.Metal Phys.8,151(1978).

[18] A.H.Kahn, J.Ruvalds, Phys.Rev.B,19,5652(1979).

[19] J.L.Birman and A.I.Solomon, Phys.Rev.Lett.49,230 (1982).

[20] J.L.Birman and A.I.Solomon,Prog.Theor.Phys.(Kyoto), Supp.80,62(1984).

[21] J.L.Birman and A.I.Solomon," State labels and selection rules in superconductors and charge-density wave superconductors", in Procedings of the XIII Colloquium on Group Theoretical Methods in Physics, ed. W.W.Zachary (World Scientific ,Singapore) 1986, p 445-9.

[22] See also the review : J.L.Birman and A.I.Solomon, "Spectrum Generating Algebras in Condensed Matter Physics" in Spectrum Generating Algebras in Physics ,ed.A.Bohm and Y.Ne'eman (World Scientific) Singapore (1986).

[23] A.I.Solomon and J.L.Birman, Phys.Lett.A88,413 (1982).

[24] A.I.Solomon and J.L. Birman, Phys.Lett.A104,235 (1984).

[25] A.I.Solomon and J.L.Birman, Phys.Lett.A111,423 (1985).

[26] A.I.Solomon and J.L.Birman, J.Math.Phys.28 ,1526(1987). 
[27] A.I.Solomon and J.L.Birman, J.Phys.C,Solid State 21, L751 (1988).

[28] S.C.Zhang, Science 275,1089 (1997); M.G.Zacher,et.al. Phys.Rev.Lett.85 , 824 (2000), and references therein.

[29] S.C.Zhang and E.Demler,Phys.Rev.B 58, 5719 (1998);see also M.Guidry,L.A.Wu,W.Sun. C.L.Wu, Phys.Rev. B63, 134516 (2001).

[30] J.Bardeen, L.N.Cooper, J.R.Schreiffer, Phys Rev. 1081175 (1957).

[31] P.W. Anderson, Phys. Rev. 112 ,1900(1958).

[32] M.Tinkham, "Superconductivity", Documents on Modern Physics, ed. E.W.Montroll, G.H.Vineyard, M.Levy, Gordon and Breach, N.Y. (1965).

[33] B.Wybourne "Classical Groups for Physicists", J.Wiley and Sons, N.Y. (1974).

[34] A. Perelomov "Generalized Coherent States and Applications" ,Springer Verlag, New York (1986).

[35] A.I.Solomon and J.L.Birman in A.Bohm and Y.Ne'eman ,eds "Dynamical Symmetry in Physics",World Science Press (1989).

[36] M.Rassetti, International Journal of Theoretical Physics 13, 425-430 (1973).

[37] J.Klauder and B-S. Skagerstrom, "Coherent States", World Science Press, Singapore (1986).

[38] W.Cochran, Phys.Rev.Lett.3, 412 (1959).

[39] P.W.Anderson, Izd.AN.SSR.,Moscow (1960).

[40] R.Cowley, Phys.Rev 134, A981(1964)

[41] M.Lines and A.Glass, "Principles and Applications of Ferroelectrics", Oxford University Press, N.Y. (1977). 
[42] M.Born and K.Huang, "Dynamical Theory of Crystal Lattices", Oxford University Press,New York (1954)

[43] See L.D.Landau and E.M.Lifshitz,"Electrodynamics of Continuous Media",Addison Wesley,MA., First Edition, (1960), p.47 and footnote on p.60.

[44] W.Cochran, Advances in Physics, 9,387 (1960); ibid. 18, 157(1969).

[45] A.D.Bruce and R.A.Cowley, J.Phys.C Solid State, 5, 595 (1972).

[46] H.Shanks, Solid State Comm.,15,753(1974).

[47] N.Sai,and D.Vanderbilt,preprint:cond-mat/0004372;

C.LaSota,C.Z.Wang,R.Yu,H.Krackauer,preprint:cond-mat/9703240.

[48] J.Kircher et.al., J.Opt.Soc.Am.B 14,705(1997).

[49] R.Henn et.al. Phys.Rev.B56,6295(1997).

[50] P.Carruthers and M.M.Nieto, Am.Jnl of Physics, 33, 537 (1965).

[51] R.J.Glauber, Phys. Rev. 231,2778(1960).

[52] J.L.Birman , Hand.der Physik, XXV/2b ,Springer Verlag ,N.Y.(1974).

[53] L.D.Landau, E.M.Lifshitz, L.P.Pitaevsky "Statistical Physics",Pergamon Press, Third Edition Oxford, (1990).

[54] K-S.Liu and M.E.Fisher,Journal of Low Temp.Phys., 10,655(1973).

[55] Y.Imry, J.Phys.C,(Solid State),8,567(1975).

[56] V.Yukevich, B.Rolov, H.E.Stanley, Ferroelectrics, 16, 61 (1977).

[57] S.V.Vonsovsky, Yu.A.Izyumov, E.Z.Kurmaev, "Superconductivity of Transition Metals", Springer-Verlag,N.Y.(1982).

[58] Yu.N.Epifanov,A.P.Levanyuk, G.M.Levanyuk, Sov.Phys.Solid State 23391 (1981). 
[59] G.L.Bir and G.E.Picus, "Symmetry and Strain-Induced Effects In Semiconductors", J.Wiley and Sons, New York (1974), Chap.IV.

[60] D.Fay and M.Weger,"Vertex Corrections in Nearly Ferroelectric Superconductors", Phys.Rev. B62,15208 (2000).

[61] M.O.Scully and M.S. Zubairy "Quantum Optics", Cambridge University Press, N.Y.(1996).

[62] F.J.Wegner, Ann.Physik, Leipzig3, 77(1994); A.Kehrein, B.Mielke, P.Neu, Zeit.Phys. B99, 269(1996).

[63] A.Leggett, A.S.Chakravarty, A.T.Dorsey, M.P.A.Fisher, A.Greg, W.Zweiger, Rev.Mod.Phys. 59, 1(1987).

[64] B.Matthias in "Ferroelectricity" ed E.Weller., Elsevier Pub.Co. (1976).

[65] J.L.Birman,Phys.Rev.Lett.17, 1216 (1966); F.E.Goldrich and J.L.Birman,Phys. Rev.167, 528 (1968); M.Weger and J.Goldberg, loc.cit..

[66] M.Weger, B.G.Silbernagel, and E.S. Greiner, Phys. Rev. Lett. 13, 521 (1964).

[67] B.M.Klein and J.L.Birman, Phys.Rev.Lett.25, 1014 (1970); Z.W.Lu, and B.M.Klein, cond-mat/9704251.

[68] D.Bloom, H.R.Shanks, J.Barnes, K.D.McKee, J.E.Ostenson, D.K.Finnemore, J.Low Temp.Phys.23,743(1976).

[69] I.E.Dzyaloshinsky,Sov.Phys.JETP 6, 621 (1957);ibid. 10 , 628 (1960);see also reference $[43] \S 28,36,40$.

[70] I.E.Dzyaloshinsky,in "Symmetries and Broken Symmetries in Condensed Matter Physics" ,ed.N.Boccara IDSET-Paris (1981).

[71] A.J.Freeman and H.Schmid "Magnetoelectric Interaction Phenomena in Crystals", Gor- 
don and Breach, New York (1975).

[72] J.L. Birman and N. Zimbovskaya,"Electrodynamics of Nearly-Ferroelectric Superconductors", (submitted for publication (March 2001)).

[73] M.L.Cohen, Phys.Rev.A134, 511(1964).

[74] A.Baratoff and G.Binnig,Physica 108B ,1335 (1981).

[75] S.I.Vedeneev ,et.al. Physica C, 198,47 (1992)

[76] J.Bardeen and D.Pines,Phys.Rev. 99, 1140 (1955); A.Fetter and A. Walecka, "Many Body Physics ”,McGraw Hill N.Y.(1974).

[77] G.M.Eliashberg,Sov.Phys.JETP, 11,696 (1960).

[78] W.L.McMillan, Phys.Rev.167, 331 (1968).

[79] R.Zeyher and G.Zwicknagl, Z.Phys.B78,175(1990).

[80] V.G.Hadjiev,et.al., Phys.Rev. B 58 , 1043 (1999).

[81] L.Pintschovius, et.al.,Europhysics Letters 37,627 (1997).

[82] M.Weger,et.al., Synth.Metals 42, 1885 (1991)

[83] V.Polinger, et.al. cond-mat/9811425.

[84] V.I.Anisimov et.al., Phys.Rev.Lett.68, 345 (1992).

[85] H.Kamimura and J.Sano, J.Supercon., 10, 279 (1997).

[86] A.S.Moskvin and Yu.D.Panov, cond-mat/9811293. 